\title{
Simple two-color array-based approach for mutation detection
}

\author{
Paolo Fortina ${ }^{1}$, Kathleen Delgrosso ${ }^{2}$, Taku Sakazume ${ }^{1}$, Rosa Santacroce ${ }^{1}$, \\ Stephane Moutereau ${ }^{1}$, Hung-Ju Su ${ }^{1,3}$, David Graves ${ }^{3}$, Steven McKenzie ${ }^{2,4}$ and \\ Saul Surrey ${ }^{2,4}$
}

${ }^{1}$ Department of Pediatrics, University of Pennsylvania School of Medicine and The Children's Hospital of Philadelphia, Philadelphia, PA; ${ }^{2}$ duPont Hospital for Children, W ilmington, DE; ${ }^{3}$ Department of Chemical Engineering University of Pennsylvania School of Engineering and Applied Science, Philadelphia, PA; ${ }^{4}$ Department of Pediatrics, Jefferson College of Medicine, Philadelphia, PA

The ability to analyze multiple polymorphic/ mutation sites rapidly and accurately is pivotal in all areas of genetic analysis. We have applied single nucleotide primer extension (SNE) for detection of multiple point mutations in a micro-array format using two-color, fluorescent dye-tagged dideoxynucleoside triphosphate terminators (ddNTPs). The oligonucleotide primer ending one nucleotide short of the mutation site being probed is bound to the slide and single-base extended in place with two different $\mathrm{Cy} 5 / \mathrm{Cy} 3$ dye-tagged terminators using solution-phase, locus-specific, single-stranded complementary templates generated by PCR from genomic DNA. The composite fluorescence produced contains peaks of distinct wave lengths corresponding to each Cy dye-tagged terminator incorporated, resulting in a fluorescent 'fingerprint' for each DNA target. DNA polymerase-catalyzed incorporation of Cy dye-tagged dideoxynucleoside triphosphates was dependent on the particular dyes, the specific ddNTP, the DNA target concentration, sequence of the template, on-slide temperature cycling and washing conditions. Results from analysis of mutations in the human hemochromatosis and connexin $\mathbf{2 6}$ genes show that this approach has several advantages over existing methods and is simple, rapid, robust, cost effective and accurate with potential applications in many areas of genetic analysis. European Journal of Human Genetics (2000) 8, 884-894.

Keywords: array-bound single nucleotide primer extension; minisequencing; fluorescent ddNTPs; mutation detection

\section{Introduction}

A variety of molecular alterations have been implicated in leading to the development of human genetic diseases and malignancies. Development of rapid, robust, parallel, highthroughput, cost-effective approaches to mutation detection are critical to generation of a thorough molecular diagnosis of life-threatening diseases. ${ }^{1}$ In addition, realization of such developments will facilitate early diagnosis and evaluation of treatment strategies.

Correspondence: Paolo Fortina MD, The Children's Hospital of Philadelphia, 310-C Abramson Pediatric Research Center, 34th Street and Civic Center Boulevard, Philadelphia, PA 19104, USA.

Tel: +12155903318; Fax: +12155903660;

E-mail: fortina@mail.med.upenn.edu

Received 8 May 2000; revised 14 July 2000; accepted 18 July 2000
A number of approaches has been used for mutation detection. ${ }^{2}$ Single-nucleotide primer extension (SNE) has been used for detection of point mutations. ${ }^{3}$ The assay is template-dependent and involves primer extension by a radioactive or dyelabeled dideoxynucleotide terminator (ddNTP) with the tag of the incorporated base revealing the identity of the template complementary nucleotide immediately $3^{\prime}$ to the primer. Solution-phase extensions followed by electrophoresis on gels as well as microtiter plate-based assays have been described. ${ }^{4-11} \mathrm{~A}$ solution-phase fluorogenic PCR 5'-nuclease or TaqM an ${ }^{\mathrm{TM}}$ assay was recently reported using seven different dyes for detection of single-nucleotide polymorphisms (SNPs) in six PCR products. ${ }^{12}$ In addition, a variety of other solution-phase mutation detection schemes 
have been described which depend on fluorescent dyes, energy transfer and/or fluorescence quenching. ${ }^{13-17}$

Chip-based extension improves on solution-phase methods since primers can be immobilized in advance on glass. Recently, a radioactive method for multiplex detection of mutations was described in which solid-phase SNE was applied to an oligonucleotide array format. ${ }^{18-20}$ In a comparison with hybridization and immobilized allele-specific probes (ASO), the power of discrimination between homozygotes and heterozygotes was one order of magnitude higher using the SNE method. Use of ASO requires at least wild-type and mutant probes ${ }^{21,22}$ with annealing and washing steps requiring extensive empirical testing to define conditions for selective removal of a single-base mismatched target. Such requirements are not optimal for production of parallel arrays that simultaneously detect multiple known point mutations in different genes. Furthermore, current chip-based ASO methods utilize four probes for each SNP call and employ photolithography to generate the chips ${ }^{23}$ which are not readily amenable to customization in a research or a clinical laboratory setting. Computer algorithms are also required to make definitive calls.

Disadvantages of current chip-based SNE approaches include use of radioisotopes or antibody conjugants for detection, and/or requirements for generation and/or purifi- cation of singlestranded target complements. In this report, we describe development of an easy, implementable, arraybased assay employing single and two-color fluorescencebased detection which has advantages over existing SNE strategies.

\section{Methods}

Leukocyte DNA from normal controls and patients harboring single-base mutationsin the hemochromatosis (HFE) ${ }^{24,25}$ and connexin $26(\mathrm{CX} 26)^{26,27}$ genes was obtained, and all genotypes were confirmed by automated DNA sequence analysis.

\section{Oligonucleotides}

Two sets of oligonucleotides (Table 1, sets A and B) were synthesized using standard phosphoramidite chemistry and purified by HPLC. Oligonucleotides to be attached to the glass surface (primer probes) were synthesized with a 5'-amino group followed by a polyethylene glycol (PEG) spacer as described previously. ${ }^{18-20}$ To conform to the evolving nomenclature for surface hybridization, we refer to such molecules as probes.

Table 1 Reagents for array-bound SNE

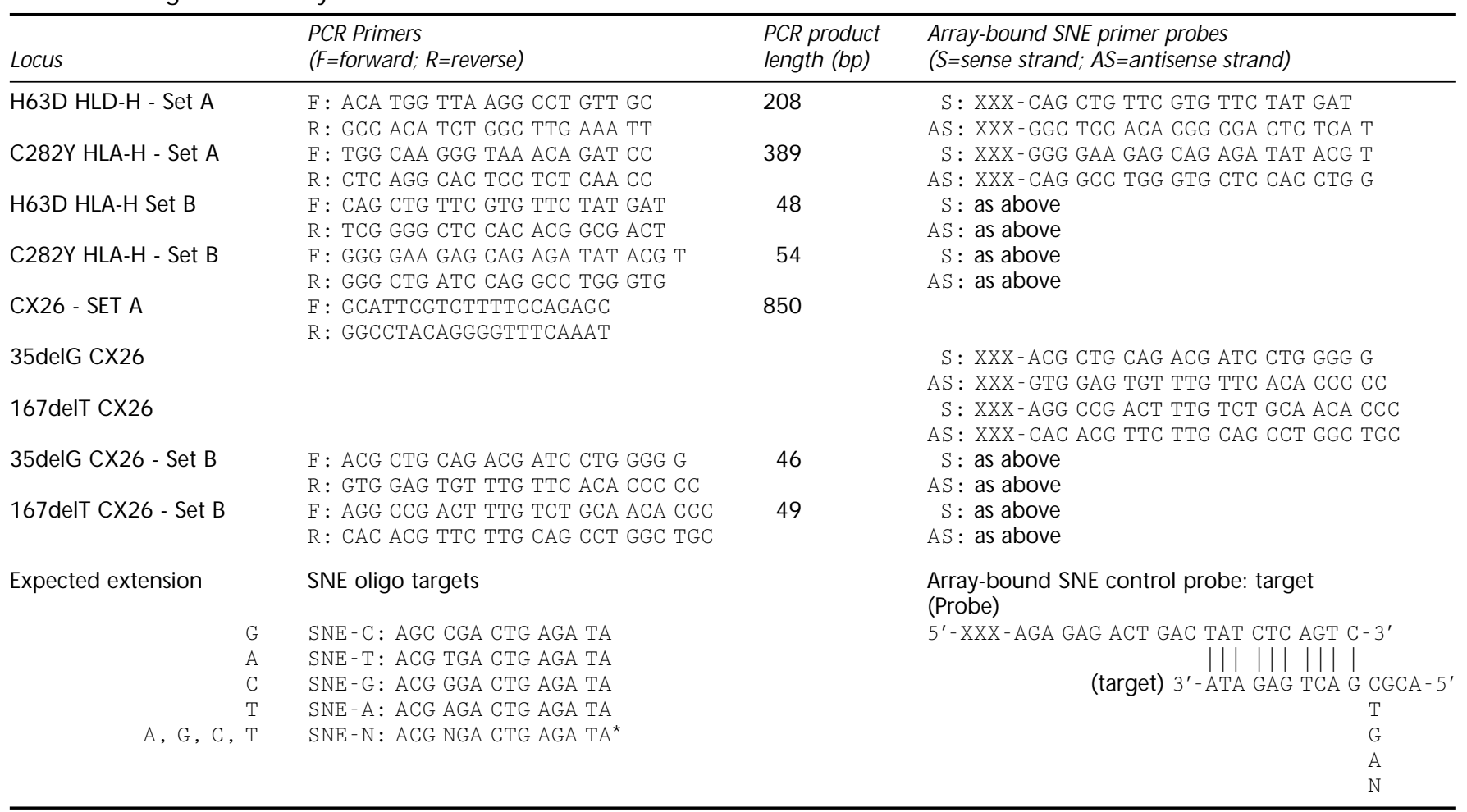

$\mathrm{XXX}=$ Aminolinker; *Can be used with any ddNTP extension since a mixture of all four nucleotides (N) was used during synthesis at this site. 


\section{SNE control}

Five oligonucleotide targets ( 14 mers) (Table 1 ) each complementary to $10 \mathrm{nt}$ at the $3^{\prime}$ end of a bound SNE oligonucleotide control primer probe ( $22 \mathrm{mer}$ ) were used as a positive target control for successful extensions on the arrays. The five targets contained either A, C, G, T or all four nucleotides located one nucleotide beyond the $3^{\prime}$ nucleotide of the bound probe, thereby facilitating array-bound probe SNE with ddTTP, ddGTP, ddCTP, ddATP or all four ddNTPs, respectively. For example, sequence of the target oligonucleotide in solution contained $A$ at this site to monitor for arraybound ddTTP extensions. Targets also contained a $3 \mathrm{nt}$ overhang beyond the site of the bound primer target/directed single nucleotide extension. Target SNE controls (12ng) are mixed with PCR products from genomic DNA prior to SNE to assess probe attachments as well as extensions with each particular terminator.

Preparation of slides Glass slides were cleaned essentially as described (http://cmgm.stanford.edu/pbrown/protocols .html).

\section{1,4-phenylene diisothiocyanate (PDC) modification and} deposition of oligonudeotide probes on slides PDC modification was as described previously. ${ }^{28}$ Poly-I-lysine slides were modified by treatment with $0.2 \%(w / v)$ PDC (Sigma, St Louis, MO, USA) in $10 \%(\mathrm{v} / \mathrm{v})$ pyridine/dimethylformamide (Fisher, Pittsburgh, PA, USA) for $2 \mathrm{~h}$ at room temperature and then washed with HPLC-grade methanol and acetone. After drying at $110^{\circ} \mathrm{C}$ for $5 \mathrm{~min}$, the PDC support was ready for attachment. The amino-modified oligonucleotides at $100 \mathrm{pmol} / \mu \mathrm{l}$ were mixed 1:1 with Micro-Spotting solution (TeleChem International Inc, Sunnyvale, CA, USA) and arrayed manually or robotically with our in-house constructed arrayer ${ }^{29}$ on to the glass supports. Remaining reactive groups on slides were blocked by incubation in $1 \mathrm{~m}$ Tris- $\mathrm{HCl}(\mathrm{pH} \mathrm{7.5)}$ for $1 \mathrm{~h}$, rinsed in $1 \mathrm{~m} \mathrm{NaCl}$ and then washed three times in $\mathrm{dd}_{2} \mathrm{O}$, twice at room temperature, followed by a final wash at $55^{\circ} \mathrm{C}$ for $15 \mathrm{~min}$. Slides were dried by centrifugation at $500 \mathrm{rpm}$ for $2 \mathrm{~min}$ in a table-top centrifuge (Sorval, Newtown, CT, USA, model RT 6000B) using a microtiter centrifuge plate holder.

\section{Generation of PCR products (targets) and array-bound single color primer extensions}

Primers were initially picked that generated PCR products of the following lengths: $208 \mathrm{bp}$ for detection of H63D and $389 \mathrm{bp}$ for detection of C282Y in the HFE gene; and $850 \mathrm{bp}$ for the entire $C X 26$ gene which can be used as target template for detection of both 35delG and 167delT mutations in that gene. PCR was performed in $50 \mu \mathrm{l}$ containing $50 \mathrm{~mm} \mathrm{KCl}$, $10 \mathrm{~mm}$ Tris- $\mathrm{HCl}(\mathrm{pH} 8.3), 1.5 \mathrm{~mm} \mathrm{M} \mathrm{gCl}_{2}, 200 \mu \mathrm{m}$ each of dATP, dCTP and dGTP, $80 \mu \mathrm{m}$ dTTP, $20 \mu \mathrm{m}$ dUTP, $100 \mathrm{ng}$ genomic DNA, 100ng each primer, 1.44 Units AmpliTaq DNA polymerase (Perkin Elmer, Applied Biosystems, Foster City, CA, USA) and 1.44 units of TaqStart antibody (Clontech, Palo
Alto, CA, USA). PCR for the two mutations in the HFE gene was multiplexed with the four primers for H63D and C282Y, while PCR of the CX26 gene was done separately. Following PCR amplification, products were treated with a mixture of $0.02 \mathrm{U} / \mu \mathrm{l}$ shrimp alkaline phosphatase (SAP) (AmershamPharmacia Biotech, Arlington Heights, IL, USA), 0.4U/ $\mu$ I E. coli exonuclease I (Exo I) (Epicenter Inc, Madison, WI, USA), and $0.02 \mathrm{U} / \mu \mathrm{l}$ uracylglycosylase (UDG) (Amersham-Pharmacia Biotech) for $60 \mathrm{~min}$ at $37^{\circ} \mathrm{C}$ and then heat inactivated at $95^{\circ} \mathrm{C}$ for $10 \mathrm{~min}$. This treatment degrades dNTPs to nucleosides, digests unincorporated primers and cleaves the PCR products at dU-containing sites. ${ }^{30}$ Fragmentation can also be accomplished by DNase I treatment ( $1 \cup$ for $15 \mathrm{~min}$ at room temperature and then heat inactivated at $65^{\circ} \mathrm{C}$ for $15 \mathrm{~min}$ ) of PCR product generated in the absence of dUTP using $200 \mu \mathrm{m}$ dTTP. Fragmented DNA (100 ng) was then mixed with ThermoSequenase buffer (Amersham-Pharmacia Biotech) and $25 \mathrm{ng}$ of SNE control oligonucleotide (Table1) in a $40 \mu \mathrm{l}$ reaction, heated to $95^{\circ} \mathrm{C}$ for $10 \mathrm{~min}$, centrifuged, cooled on ice and pipetted on to the array. The solution was incubated for $5 \mathrm{~min}$ at room temperature to facilitate probe/target annealing, and then $10 \mu \mathrm{l}$ of a mixture containing $1 \mu \mathrm{l}$ of $40 \mu \mathrm{m}$ non-competitive ddNTPs, $1 \mu \mathrm{l}$ of $40 \mu \mathrm{m}$ Cy5 ddNTP, 6.4 units ThermoSequenase (Amersham-Pharmacia Biotech), in 1X ThermoSequenase buffer was added to the array. The array was fitted with a cover slip and incubated for $20 \mathrm{~min}$ at $48^{\circ} \mathrm{C}$. After incubation the array was washed in $55^{\circ} \mathrm{C} \mathrm{ddH}_{2} \mathrm{O}$ for $5 \mathrm{~min}$, dried and then scanned.

\section{Two-color array-bound primer extensions}

More recently, we have generated much shorter PCR products by choosing primer pairs immediately flanking the mutation site being interrogated (Table1, set $\mathrm{B}$ ). This results in much smaller PCR products (45-50 bp) and eliminates the need for dU-mediated cleavage step with UDG. PCR was performed in a $50 \mu \mathrm{l}$ reaction containing $50 \mathrm{~mm} \mathrm{KCl}, 10 \mathrm{~mm}$ Tris- $\mathrm{HCl}$ (pH 8.3), 2.0mm $\mathrm{MgCl}_{2}, 200 \mu \mathrm{m}$ each dNTP, 100ng DNA, $100 \mathrm{ng}$ each primer, 1.44 units AmpliTaq DNA polymerase and 1.44 units of TaqStart antibody. PCR for monitoring the two mutations in the HFE gene was multiplexed using four primers flanking the $\mathrm{H} 63 \mathrm{D}$ and $\mathrm{C} 282 \mathrm{Y}$ mutations. PCRs for CX-26 mutations were done separately because of proximity of the two mutations which, if multiplexed, could result in generation of a longer PCR product by using one forward and one reverse primer from the two sites being probed for the mutations. Conditions for PCR for HFE and CX26 mutations were $95^{\circ} \mathrm{C}$ for $5 \mathrm{~min}$ followed by $10 \mathrm{cycles}$ at $94^{\circ} \mathrm{C}$ for $15 \mathrm{~s}$, $70^{\circ} \mathrm{C}$ for $5 \mathrm{~s}$ decreasing the temperature $1^{\circ} \mathrm{C}$ per cycle to $60^{\circ} \mathrm{C}$, then $72^{\circ} \mathrm{C}$ for $10 \mathrm{~s}$ followed by $22 \mathrm{cycles}$ at $94^{\circ} \mathrm{C}$ for $15 \mathrm{~s}, 60^{\circ} \mathrm{C}$ for $5 \mathrm{~s}$ and $72^{\circ} \mathrm{C}$ for $10 \mathrm{~s}$. This protocol eliminates formation of primer dimers that can co-migrate with the desired PCR products making quantitation difficult. Primer dimer formation was monitored by running a PCR reaction in the absence of genomic DNA. The PCR reaction was treated with a 
mixture of SAP and Exol for $60 \mathrm{~min}$ at $37^{\circ} \mathrm{C}$ and heat inactivated at $95^{\circ} \mathrm{C}$ for $10 \mathrm{~min}$.

Two-color SNE experiments using the smaller PCR products were performed as follows: $30 \mathrm{ng}$ of PCR product was mixed with ThermoSequenase buffer, $12 \mathrm{ng}$ of SNE control oligonucleotide, $1 \mu \mathrm{l}$ of $40 \mu \mathrm{m}$ non-competitive ddNTP, $1 \mu \mathrm{l}$ of $40 \mu \mathrm{m}$ Cy5 ddNTP, $4 \mu \mathrm{l}$ of $40 \mu \mathrm{m}$ Сy3 ddNTP for ddG and ddTTP or a final concentration of $7.5 \mu \mathrm{m}$ ddCTP or $16 \mu \mathrm{M}$ ddATP, $1 \mu \mathrm{l}$ of $10 \mu \mathrm{g} / \mu \mathrm{l}$ BSA, $25 \mu \mathrm{l} 2 \times$ Self-Seal Reagent (MJ Research, Inc., Waltham, MA, USA) and 32 units ThermoSequenase in a $50 \mu \mathrm{l}$ reaction. The reaction mixture was pipetted on to the array and covered with a cover slip. The array was then placed on to the heating block of a PerkinElmer 9600 Thermocycler and SNE performed using a PCR cycling protocol. On-slide temperature cycling was done as follows: $96^{\circ} \mathrm{C}$ for $15 \mathrm{~s}, 50^{\circ} \mathrm{C}$ for $10 \mathrm{~s}$, and $72^{\circ} \mathrm{C}$ for $20 \mathrm{~min}$ for four cycles, after which the slide was placed in a slide holder in a $62^{\circ} \mathrm{C} \mathrm{ddH_{2 }}$ O bath for $5 \mathrm{~min}$. Slides were dried and then scanned.

\section{ScanArray $\mathbf{3 0 0 0}$ protocols and settings}

A ScanArray 3000 (GSI Lumonics Inc, Watertown, MA, USA) was used to monitor $\mathrm{Cy} 5$ as well as $\mathrm{Cy} 3$ fluorescence using a laser power setting of $100 \%$ and PMT value of $100 \%$. Extension signals for Cy5-tagged terminator are sometimes off-scale on the scanner necessitating a decreased setting for the laser from 100 to $75 \%$ in order to obtain data which is on the linear portion of scanner response (0-65000 gray scale units, linearity of scanner response for Cy 5 -terminator is between approximately 5-5000 attomoles deposited in spot area of about $1 \times 10^{6} \mu^{2}$ ). Cy3 extensions were 2 - to 3-fold less than Cy5 using about $1 \mu \mathrm{m}$ terminator final concentrations. Scans of a dilution series of the 2 dye-tagged terminators at $100 \%$ settings for laser and photomultiplier as a function of attomoles of each dye deposited shows about a 2.7-fold higher reading for the same amount of terminator when comparing Cy5 to Сy3 (data not shown). This is consistent with a higher molar extinction coefficient and/or quantum yield for Cy5 compared with Cy3. In fact, the ratio of molar extinction coefficients for Cy5/Cy3-bound protein conjugates is $250000 / 150000$ or 1.67 , and the ratio of quantum yields is $0.28 / 0.15$ or $1.87 . .^{31}$ The product of these two ratios is 3.13 which approximates observed differences in Cy5/Cy3 fluorescence when spotting equimolar amounts of the two dyes on arrays.

Source of dyelabeled dideoxynucleotides Nucleotides labeled with $\mathrm{Cy} 3$ and $\mathrm{Cy} 5$ dyes (Amersham-Pharmacia Biotech) were prepared using C5 (pyrimidine) or C7 (purine) modified nucleotides. $^{32}$

\section{Results}

\section{Multiplex, single-color SNE}

The basic strategy for array-bound SNE is shown in Figurel. Expected array-bound extensions ( + ) with each of the four
ddNTPs are seen on array-bound sense and antisense oligoprobes for the 16 possible 2-allele combinations (Figure2, panel A). We first explored multiplexing so that simultaneous PCR and SNE using Cy5-tagged terminators could be implemented for detection of two different mutations on the same array. Expected ddNTP extensions for all nine possible genotypes at the two loci in the HFE gene using array-bound sense and antisense primers are shown in Figure2, panel B. Multiplex PCR of human genomic DNA to screen for the two changes causing hemochromatosis (HFE) ${ }^{24,25}$ is shown in Figure2, panel C. PCR reactions contained two separate primer pairs (total of four in one reaction) which resulted in simultaneous PCR of the two different HFE regions. The PCR products were then interrogated for the two mutations (H63D and C282Y) each of which results in an amino acid change. The array contained $\mathrm{H} 63 \mathrm{D}$ and $\mathrm{C} 282 \mathrm{Y}$ sense and antisense bound primers as well as an SNE-bound control oligonucleotide primer. The SNE control lights up on all arrays since it represents an oligoprimer that can be extended by all four dye-tagged ddNTPs when annealed to its target complement oligonucleotide added to the PCR target solution just prior to SNE. Expected extensions (black circles) are shown in diagram form on the left in panel C. Extension on the array was done with Cy5-ddGTP providing a positive signal for H63D antisense and C282Y sense rows on the array. Genotype of the patient, determined by DNA sequence analysis was homozygous normal at the H63D (C on the sense strand) and heterozygous at the $C 282 \mathrm{Y} \mathrm{loci}$ ( $G$ and $A$ on the sense strand). Average florescence intensities of the row of four spots for each primer are in parentheses. These results show robust signals using Cy5-ddGTP extension for both H63D (normal/normal) as well as for C282Y (normal/ mutant), consistent with presence of a normal allele at both loci. Since both sense and antisense SNE primers are array bound, heterozygote detection using the same dye for all four ddNTPs requires a second separate array extension with one of the three remaining ddNTPs.

We next typed five different genomic DNA samples for the same two mutations and compared those results with that expected from DNA analysis. Expected and observed results are shown in Table2 for Cy5-ddGTP array-bound extensions from five different individuals (eg samples001 to 005). Multiplex PCRs were done with two sets of primer pairs in five separate reactions, one of each of the two loci in the five individuals, and PCR products were incubated on five separate arrays to si multan eously assess these HFE genotypes. Averages for the scanning of the four quadruplicate spots representing sense and antisense primer registers at the $H 63 D$ and C282Y loci are shown with results at each of the four spots. An SNE control is also included in quadruplicate on each array that extended with any dye-tagged terminator. Comparison of signals at the sense and antisense registers for the two HFE sites shows correct extensions for all five individuals characterized by a 5-10-fold signal to noise ratio (eg positive/negative average signals at sense and antisense 
A

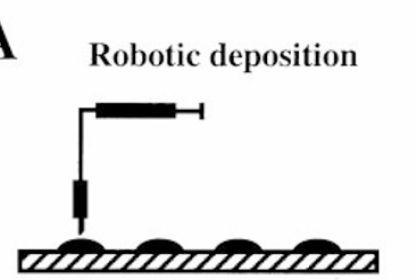

Amino-silanized polylysine-PDC treated glass slide

E

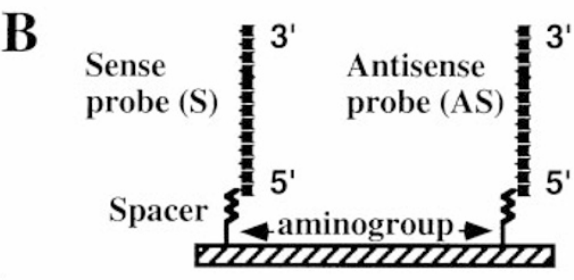

F

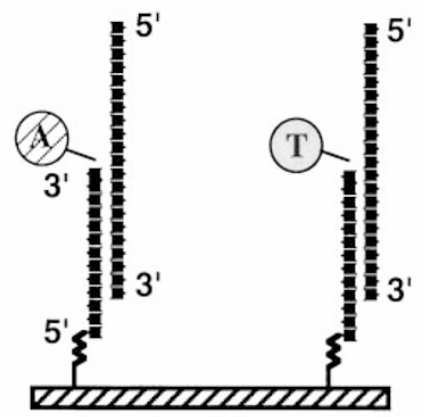

DNA polymerase + dye-tagged ddNTP

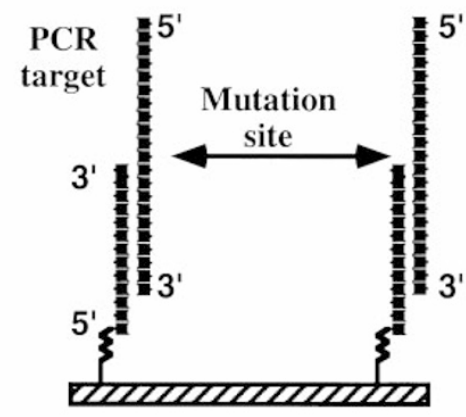

Probe : Target
C

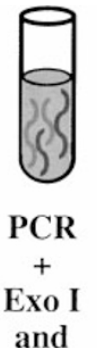

Alk Phos

treatment

(+/- UDG)
D

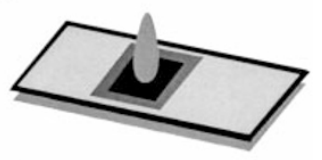

SNE reaction mix

Extension

Washing

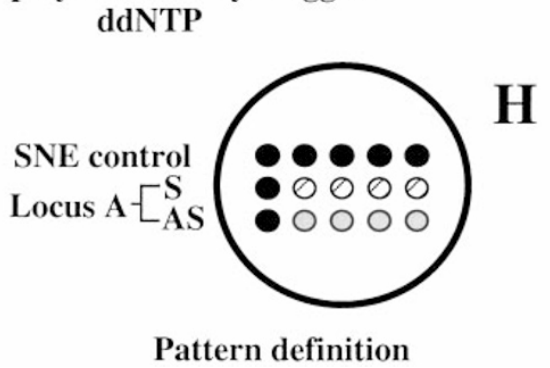

Pattern definition

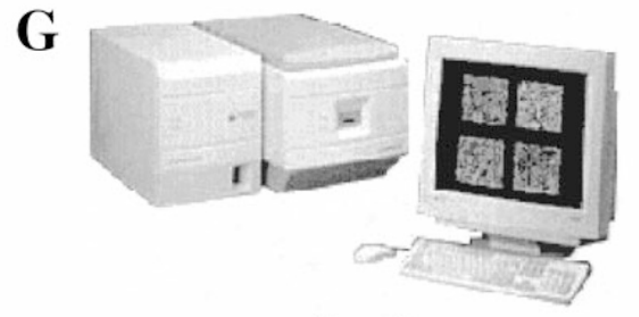

Scanning

Figure 1 Strategy for array-bound SNE. Sense and antisense oligonucleotide probes containing 5' spacer and aminogroup are attached to amino-silanized polylysine-PDC treated glass slide following manual or robotic deposition (A and B). PCR targets are generated from genomic DNA and treated with Exol and SAP (C). (Longer PCR products are synthesized in the presence of dUTP and subsequently cleaved with UDG). PCR targets are then placed on a slide containing the bound oligoprobes and SNE performed (D). After annealing of the complementary PCR target strand to the bound oligoprobes (E), a single dye terminator extension occurs (F) which is then monitored by scanning (G) giving rise to a specific pattern of fluorescence $(\mathbf{H})$.

registers) for individuals with $\mathrm{N} / \mathrm{N}$ genotypes at either locus.

\section{Two-color SNE}

Two-color multiplex extensions with Cy5-ddCTP and Cy3-ddTTP are shown in Figure3. In this experiment we simultaneously probed on the same array for two common singlenucleotide deletions in the connexin 26 (CX26) gene ${ }^{26,27}$ using a mixture of Cy5-ddCTP and Cy3-ddTTP. Again, signals are seen for both dyes at the proper array registers. Signals for Cy3 extensions were 2- to 3-fold lower than Cy5.

\section{Two-color SNE using short PCR products, increased Cy3-ddNTPs concentration, and on-slide temperature}

cycling We next designed primers to generate much smaller PCR products (40-60 bp) in order to el iminate requirements for fragmentation of PCR fragments prior to array-bound SNE. In addition, concentration of the Cy3 ddNTPs was increased into an effort to maximize SNE with these terminators. Finally, an on-slide temperature cycling approach was also employed in an effort to further increase array-bound extensions for both Cy5 and Cy3 ddNTPs. Robust signals are now evident for both Cy5 and Cy3 extensions without the need for PCR fragmentation (Figure 4). In this experiment the ratio of concentrations for $\mathrm{Cy} 3 / \mathrm{Cy} 5$ terminators was about 20/1 (16 $\mu \mathrm{m}$ compared with $0.75 \mu \mathrm{m})$. On-slide temperature cycling also appears to increase extensions for both dyetagged terminators. 
A

\begin{tabular}{|ll|lllll|llll|}
\hline Allele & \multicolumn{5}{|c|}{$\begin{array}{l}\text { Sense } \\
\text { oligoprobe }\end{array}$} & \multicolumn{5}{c|}{$\begin{array}{l}\text { Antisense } \\
\text { oligoprobe }\end{array}$} \\
\hline $\mathbf{1}$ & $\mathbf{2}$ & A & G & C & T & T & C & G & A \\
\hline A & A & + & - & - & - & + & - & - & - \\
A & G & + & + & - & - & + & + & - & - \\
A & C & + & - & + & - & + & - & + & - \\
A & T & + & - & - & + & + & - & - & + \\
\hline G & A & + & + & - & - & + & + & - & - \\
G & G & - & + & - & - & - & + & - & - \\
G & C & - & + & + & - & - & + & + & - \\
G & T & - & + & - & + & - & + & - & + \\
\hline C & A & + & - & + & - & + & - & + & - \\
C & G & - & + & + & - & - & + & + & - \\
C & C & - & - & + & - & - & - & + & - \\
C & T & - & - & + & + & - & - & + & + \\
\hline T & A & + & - & - & + & + & - & - & + \\
T & G & - & + & - & + & - & + & - & + \\
T & C & - & - & + & + & - & - & + & + \\
T & T & - & - & - & + & - & - & - & + \\
\hline
\end{tabular}

C

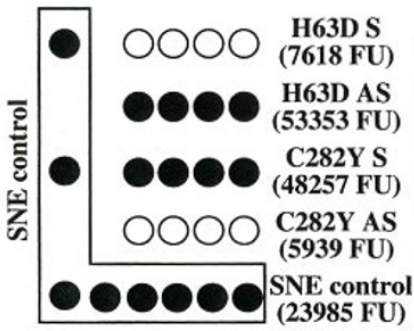

: expected signal
B Expected ddNTP extensions for all 9 possible genotypes at two loci (C282Y and H63D) on array-bound sense (S) and antisense (AS) SNE primers

\begin{tabular}{|lll|lll|}
\hline \multicolumn{3}{|c|}{ C282Y (G to A) } & \multicolumn{3}{c|}{ H63D (C to G) } \\
Genotype & S & AS & Genotype & S & AS \\
\hline N/N & G & C & N/N & C & G \\
N/N & G & C & N/M & C, G & G, C \\
N/N & G & C & M/M & G & C \\
N/M & G, A & C, T & N/N & C & G \\
N/M & G, A & C, T & N/M & C, G & G, C \\
N/M & G, A & C, T & M/M & G & C \\
M/M & A & T & N/N & C & G \\
M/M & A & T & N/M & C, G & G, C \\
M/M & A & T & M/M & G & C \\
\hline
\end{tabular}

\section{Cy5 ddGTP}

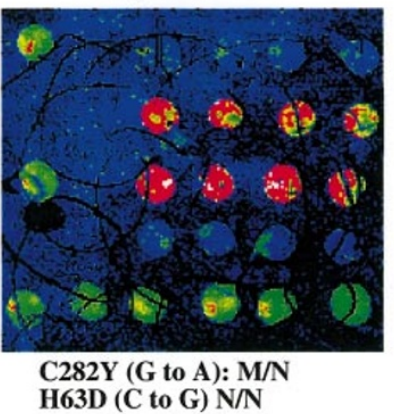

Figure 2 Detection of hemochromatosis mutations using single color SNE. Expected ddNTP extensions ( +) on array-bound sense and antisense oligoprobes for each of the 16 possible nucleotide combinations for two alleles are shown in panel A. Expected extensions on sense (S) and antisense (AS) bound probes for nine possible genotypes in the HFE gene at the C282Y and H63D sites is shown in panel B. Arrays containing bound sense and antisense primers (Table 1) were extended as described in Methods with Cy5-ddGTP using two UDG-fragmented PCR products which encompass the sites of two mutations in the HFE gene (H63D and C282Y) (panel C). The two PCR reactions were multiplexed in the same tube and incubated on the same array. Expected extensions are indicated (dark circles in panel C) with experimental values shown for fluorescence (FU) representing the average of the four spots in each row shown in color on array scan.

Expected two-color SNE extensions based on DNA sequence analysis were observed for the two loci interrogated allowing unambiguous typing of N/M at $\mathrm{C} 282 \mathrm{Y}$ and N/N at $\mathrm{H} 63 \mathrm{D}$. For example, at the $\mathrm{C} 282 \mathrm{Y}$ locus, extension occurred with Cy3-ddATP on the sense strand bound primer indicating presence of a mutant allele ( $G$ to $A$ on the sense strand). In addition, extension occurred with Cy5-ddCTP on the antisense bound primer also indicating presence of a normal allele ( $G$ on the sense strand). Hence, typing at this locus shows heterozygosity for both normal and mutant alleles. Results for the H63D locus show presence of two normal alleles since extension occurred with Cy5-ddCTP on the sense strand indicating presence of one or two normal alleles (C on sense strand). Homozygosity for the normal allele is inferred since no extension with Cy5-ddCTP is detected on the antisense strand bound primer. Presence of the mutant allele ( $C$ to $G$ on the sense strand) would have resulted in extension with ddCTP on the antisense bound primer. No extensions at the H63D locus were expected using Cy3-ddATP and none were observed.

On-slide temperature cycling increased both Cy5 and Cy3 extensions comparing one against three cycles. In these 
Table 2 PCR detection of HFE mutations in five individuals using single color SNE (Cy5-ddGTP extension)

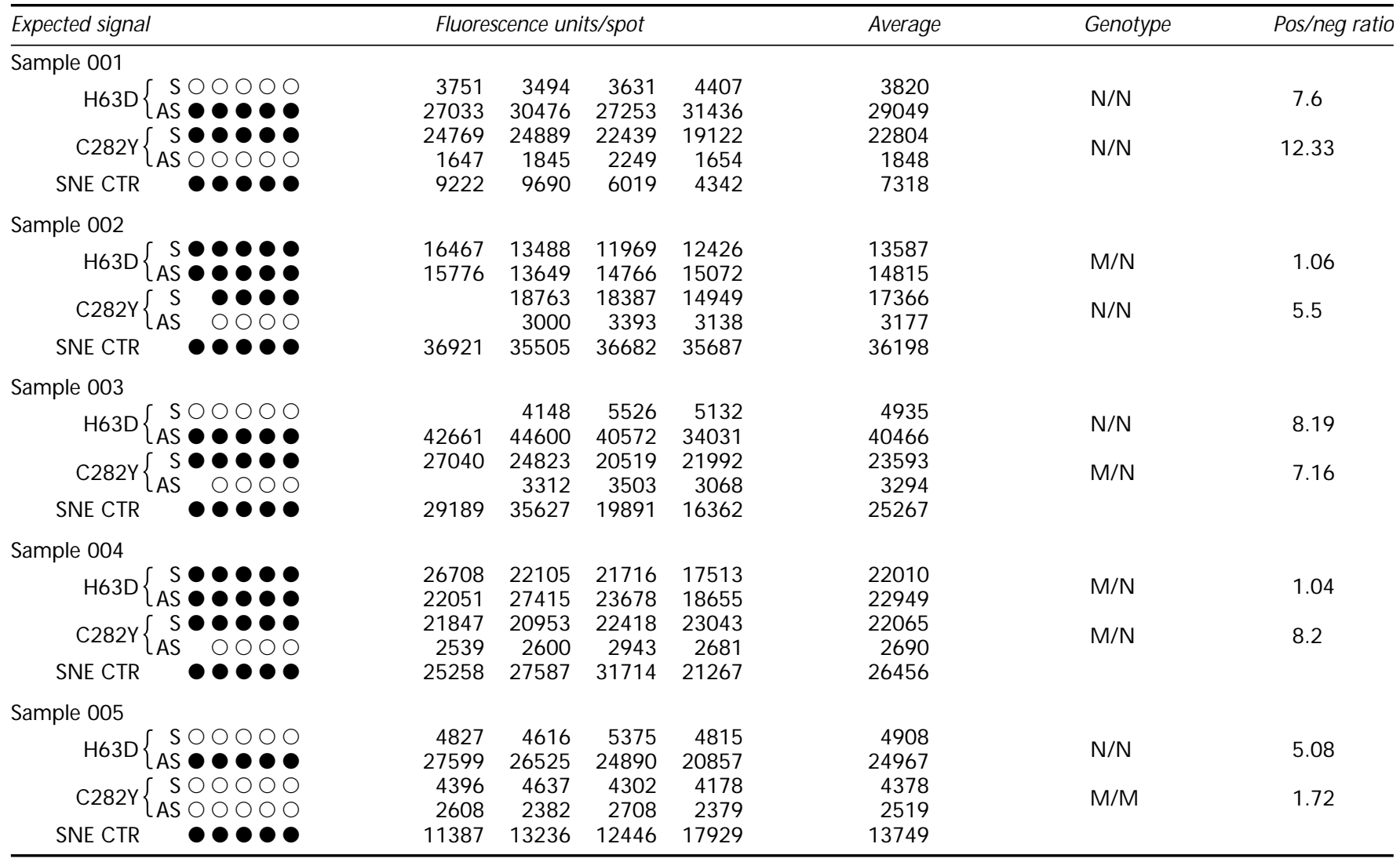
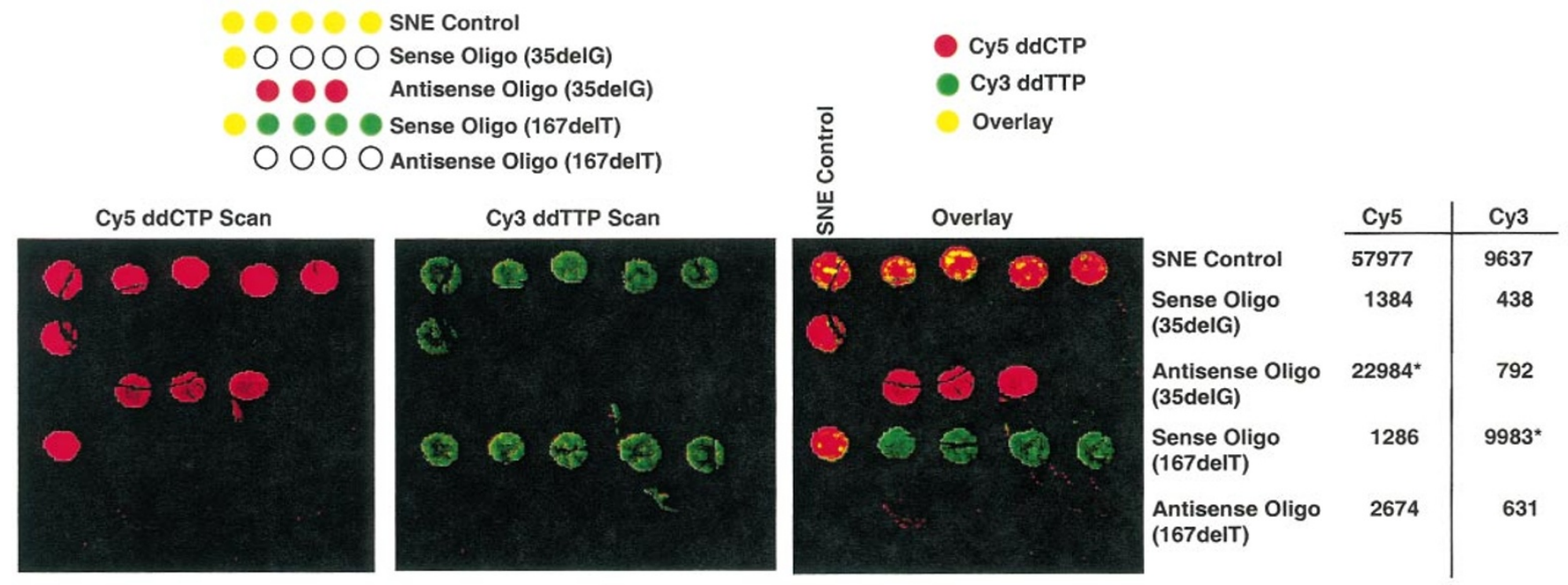

Figure 3 Detection of connexin 26 mutations using 2-color SNE. Processed images of scans are shown for Cy5 (left panel), Cy3 (center panel) and overlay of both Cy5 and Cy3 (right panel) SNE extensions on the same array. Note SNE controls (top row and three spots in left column of each panel in Cy5 and Cy3 scans) extend with both dyes generating a yellow to orange color in the overlay. Schematic diagram at top illustrates expected results for the overlay. Table on right shows averages for Cy5 and Cy3 extensions for each row; * indicates expected extensions. 
A

$\begin{aligned} & \text { SNE control } \\ & \text { (56659 FU) }\end{aligned}$
H63D sense*
(42257 FU)
H63D antisense
(396 FU)
C282Y sense
(129 FU)
C282Y antisense*
(16161 FU)
(16161 FU)

B

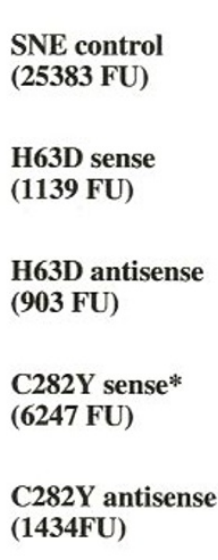

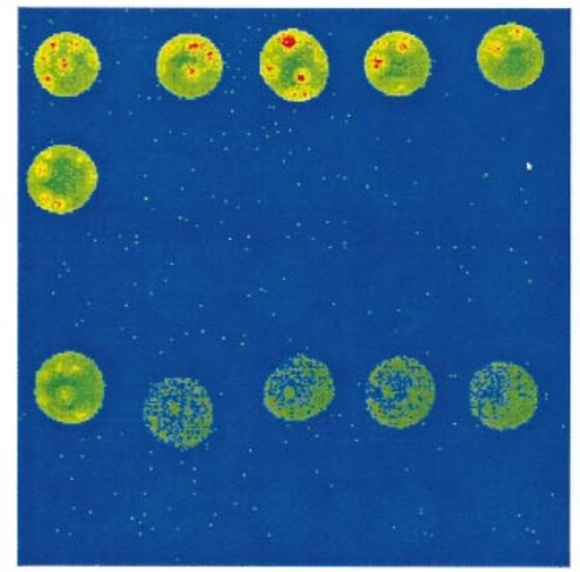

1 cycle

Cy5-ddCTP Extensions

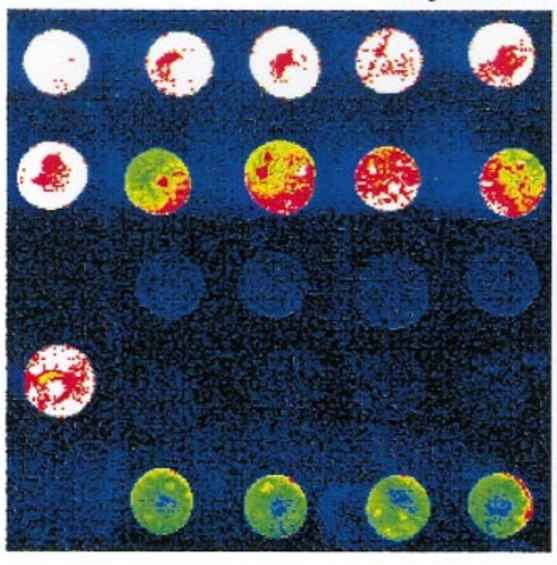

1 cycle

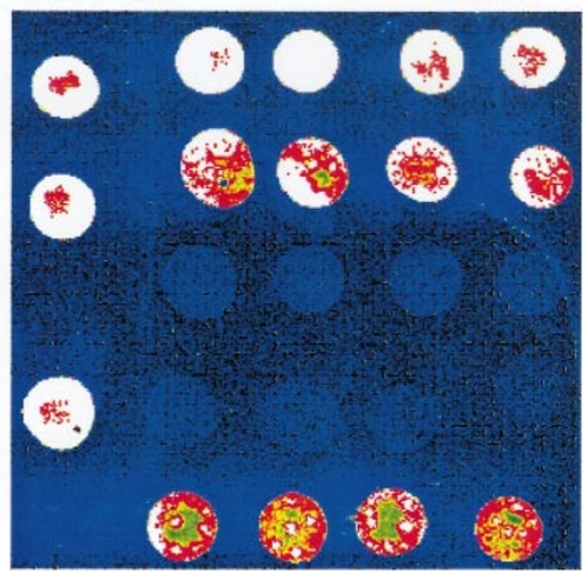

3 cycles
SNE control (57131 FU)

H63D sense* (52234 FU)

H63D antisense (564 FU)

C282Y sense (404 FU)

C282Y antisense* (43689 FU)

Figure 4 Detection of hemochromatosis mutations using 2-color SNE and on-slide temperature cycling. Arrays containing bound sense and antisense primers were extended as described in Methods with Cy5-ddCTP $(0.75 \mu \mathrm{m})$ and Cy3-ddATP (16 $\mu \mathrm{m})$ using small PCR products (40-60 bp, Table3) which encompass the H63D and C282Y mutations in the HFE gene. The two PCR reactions were multiplexed in the same tube and incubated on the same array; * expected extensions with the two different dye-tagged terminators with values for fluorescence (FU) representing the average of the four spots in each row. Scans for Cy5 (panel A) and Cy3 (panel B) for one cycle (left frames) and three cycles (right frames) of on-slide temperature cycling were as described in Methods. Slides were scanned and quantitated using a ScanArray 3000 . SNE controls (top row and three spots in far left column of each frame in panels A and B) extend with both ddNTPS.

experiments, arrays were done in parallel on the same day using Cy5 and Cy3 extensions on the same array. The SNE FU controls for Cy5 extensions were almost identical for one (56659) and three (57131) cycles, whilst values for Cy5 extensions at one against three cycles increased from 42257 to 52234 for one locus (H63D) and from 16161 to 43689 for the second locus (C282Y). Values for Cy3 extensions at C282Y were 6247 and 22555 with SNE FU controls for Cy3 extensions of 25383 and 40856 at one against three cycles, respectively.

\section{Discussion}

Our strategy for two-color array-based detection of mutations by single-nucleotide primer extension offers numerous advantages over existing strategies: 
(1) Cy5/Cy3 dye-tagged ddNTPs are employed instead of fluorescein, which is subject to photobleaching; ${ }^{32}$

(2) on-slide temperature cycling increases signal intensity maximizing extensions for robust SNE;

(3) use of an SNE control which extends with any of the terminators represents an internal control for attachment, annealing and SNE on each array;

(4) primers designed to generate 40 to $60 \mathrm{bp}$ PCR products eliminate the need for fragmentation prior to SNE;

(5) the assay is robust and straightforward and does not require generation or purification of singlestranded targets or antibody conjugants for detection;

(6) the entire assay is greatly simplified since PCR reactions followed by alkaline phosphatase and exonuclease I digestion are done in the same tube, and then the entire tube contents are directly applied to the array;

(7) if long PCR products are needed, dUTP can be used during PCR followed by UDG cleavage to generate optimal targets for SNE;

(8) the use of short PCR products may eliminate misincorporation due to generation of secondary structures in templates which can form in long PCR products; $^{33}$

(9) a direct read-out of Cy5/Cy3 fluorescence is obtained on a commercially available fluorescence scanner, since computer-based algorithms like those for evaluation of ASO-based arrays are not required because direct read-out is apparent for normal and mutant alleles without use of antibody conjugants or radioisotopes; and

(10) the system is readily implementable in research and clinical laboratories.

Our results suggest $\mathrm{Cy} 3$ extensions are less efficient than Cy5, and that different dyes may inhibit polymerase-catalyzed extension to different degrees. This conclusion is further substantiated by the much lower level of Cy3 extensions compared with Cy5 for the C282Y locus (ie see Figure3) when using similar ddNTPs concentrations on the same array. For example, robust Cy5 extensions were evident using $0.75 \mu \mathrm{m}$ ddNTPs, whilst robust Cy3 extensions require a much higher concentration of ddNTPs. Furthermore, for Cy3 extensions there was also a preference for incorporation of the different ddNTPs ( $\mathrm{G}>\mathrm{T}>\mathrm{C}>\mathrm{A}$, data not shown). In addition, our results suggest that extension efficiencies vary with the same dye-tagged terminator depending on what locus is being interrogated, suggesting a preference for certain primer-template configurations.
Although this approach is ideal for typing known SNPs or mutations, its utility defining new mutations in continuous DNA sequence analysis remains unexplored. One of the major cost implications of array-based SNE for sequencing is the cost of the array-bound oligonucleotide primers which traditionally contain a spacer arm and an aminolink group and have required HPLC purification. Our recent experiences indicate that HPLC purification may not be required for capture of complementary targets by array-bound probes (S McKenzie et al, 1999 unpublished observations). In addition, spacer-containing sense and antisense primers ending one nucleotide short of the base being interrogated and which are used for array-bound SNE can also be used as forward and reverse primers in solution-phase to generate the short PCR products (data not shown), thereby minimizing oligonucleotide cost. Costs could be decreased further by direct attachment of spacers to the array surface instead of the more commonly used costly spacer synthesis directly on the oligonucleotide primers. $^{34}$ A two-color array-based approach decreases the number of arrays from four to two for typing unknown nucleotide changes, in addition to the elimination of the need for use of radioisotopes. In addition, obvious savings are realized when scale up is achieved, since maximization of the number of loci to be interrogated simultaneously using a multicolor fluorescence approach will minimize the cost of having to use multiple arrays to obtain the same information.

The development of a robust four-color SNE protocol would eliminate the need to do two independent arrays now required with a two-color approach we describe to type each allele, if the base change is not known. This would require use of four different dyes the spectral properties of which for excitation and emission were appropriate to accomplish the four-color simultaneous SNE. Such dye-labeled terminators are currently used for automated DNA sequence analysis but are not commercially sold separately. Existing dyes can be chemically attached to terminators to generate these substrates but are very costly, therefore limiting their use. In addition, with a simultaneous four-color approach only one bound oligonucleotide primer would be required to interrogate each locus. In fact, preliminary studies show feasibility for four-color detection on arrays using arrayed primer extension (APEX) methodology. ${ }^{35-40}$ However, in cases where the normal and mutant polymorphic site are known, then only one two-color array needs to be done to type unambiguously the particular site.

In summary, we describe a two-color array-based approach to mutation detection using Cy5-/Cy3-dye labeled ddNTPs which has a number of important advantages over existing SNE-based approaches. Multicolor array-bound SNE will be of major advantage provided that the dyelabeled terminators become commercially available at a reasonable cost and that the number of sites which can be interrogated on a single array is high, thereby maximizing allele calls and minimizing cost. 


\section{Acknowledgements}

We wish to thank Drs P Gasparini and M Carella, IRCCS Casa Sollievo della Sofferenza, San Giovanni Rotondo, Foggia, and Drs C Camaschella and A Roetto, Dipartimento di Scienze Biomediche e Oncologia Umana, Universita di Torino, Italy, for providing samples, and Drs C Fuller and J Nelson, Amersham-Pharmacia Biotech, Piscataway, NJ, USA, for hel pful and valuable suggestions and insights on using $\mathrm{Cy}$-labeled ddNTPs. This work was supported in part by NIH grants P60-HL38632, P30-HG00425 (PF, DG), by the Nemours Foundation (S M CK and SS) and CNR Target Project on Biotechnology (PF).

\section{References}

1 McKenzieS, Mansfield E, Rappaport E, Surrey S, Fortina P: Parallel molecular genetic analysis. Eur J Hum Genet 1998; 6: 417-429.

2 Landegren $U$, Nilsson M, Kwok P-Y: Reading bits of genetic information: methods for single-nucleotide polymorphism analysis. Genome Res 1998; 8: 769-776.

3 Syvanen A-N: From gels to chips: 'Minisequencing' primer extension for analysis of point mutations and single nucleotide polymorphisms. Hum Mutat 1999; 13: 1-10.

4 Syvänen A-C, Aalto-Setälä K, Harju L, Kontula K, Söderlund H: A primer-guided nucleotide incorporation assay in the genotyping of apolipoprotein E. Genomics 1990; 8: 684-692.

5 Kuppuswamy MN, Hoffmann JW, Kasper CK, Spitzer SG, Groce SL, Bajaj SP: Single nucleotide primer extension to detect genetic diseases: Experimental application to hemophilia B (factor IX.), cystic fibrosis genes. Proc Natl Acad Sci USA 1991; 88: 1143-1147.

6 Kobayashi M, Rappaport E, Blasband A et al: Fluorescence-based DNA minisequence analysis for detection of single-base changes in genomic DNA. Mol Cell Probes 1995; 9: 175-182.

7 Fahy E, Nazarbaghi R, Zomorrodi $M$ et al: Multiplex fluorescencebased primer extension method for quantitative mutation analysis of mitochondrial DNA and its diagnostic application for Alzheimer's disease. Nucleic Acids Res 1997; 25: 3102-3109.

8 Syvänen A-C, Ikonen E, Manninen T et al: Convenient, quantitative determination of the frequency of a mutant allele using solidphase minisequencing: application to aspartylglucosaminuria in Finland. Genomics 1992; 12: 590-595.

9 Jalanko A, Kere J, Savilhati E et al: Screening for defined cystic fibrosis mutations by solid-phase minisequencing. Clin Chem 1992; 38: 39-43.

10 Livak KJ, Hainer JW: A microtiter plate assay for determining apolipoprotein E genotype, discovery of a rare allele. Hum Mutat 1994; 3: 379-385.

11 Nikiforov TT, Rendle RB, Goelet P et al: Genetic bit analysis: a solid phase method for typing single nucleotide polymorphisms. Nucleic Acids Res 1994; 22: 4167-4175.

12 Lee LG, Livak KJ, Mullah B, Graham RJ, Vinayak RS, Woudenberg TM: Seven-color, homogeneous detection of six PCR products. Biotechniques 1999; 27: 342-349.

13 Nazarenko IA, Bhatnagar SK, Hohman RJ: A closed tube format for amplification and detection of DNA based on energy transfer. Nucleic Acids Res 1997; 25: 2516-2521.

14 Chen X, Kwok P-Y: Template directed dyeterminator incorporation (TDI) assay: a homogeneous DNA diagnostic method based on fluorescence resonance energy transfer. Nucleic Acids Res 1997; 25: 347-353.

15 Bernard PS, Ajioka RS, Kushner JP, Wittwer CT: Homogeneous multiplex genotyping of hemochromatosis mutations with fluorescent hybridization probes. Am J Pathol 1998; 153: 1055-1061.
16 Chen X, Livak KJ, Kwok P-Y: A homogeneous, ligase-mediated DNA diagnostic test. Genome Res 1998; 8: 549-556.

17 Tyagi S, Kramer FR: M olecular beacons: probes that fluoresce upon hybridization. Nat Biotechnol 1996; 14: 303-308.

18 Shumaker JM, Metspalu A, Caskey CT: Mutation detection by solid phase primer extension. Hum Mutat 1996; 7: 346-354.

19 Pastinen T, Kurg A, Metspalu A, Peltonen L, Syvanen A-C: Minisequencing: a specific tool for DNA analysis and diagnostics on oligonucleotide arrays. Genome Res 1997; 7: 606-614.

20 Pastinen T, Perola M, Niini P et al: Array-based multiplex analysis of candidate genes reveals two independent and additive genetic risk factors for myocardial infarction in the Finnish population. Hum Mol Genet 1998; 7: 1453-1462.

21 Wallace RB, Johnson MJ, Hirose T, Miyake T, Kawashima EH, Itakura K: The use of synthetic oligonucleotides as hybridization probes. II. Hybridization of oligonucleotides of mixed sequence to rabbit beta-globin DNA. Nucleic Acids Res 1981; 9: 879-894.

22 Conner BJ, Reyes AA, Morin C, Itakura K, Teplitz RL, Wallace RB: Detection of sickle cell $\beta^{\text {s}}$-globin allele by hybridization with synthetic oligonucleotides. Proc Natl Acad Sci USA 1983; 80: 278-282.

23 Wang DG, Fan JB, Siao CJ et al: Large-scale identification, mapping, and genotyping of single-nucleotide polymorphisms in the human genome. Science 1998; 280: 1077-1082.

24 Beutler E, Gelbart T, West C et al: Mutation analysis in hereditary hemochromatosis. Blood Cells Mol Dis 1996; 22: 187-194.

25 Carella M, D'Ambrosio L, Totaro A: Mutation analysis of the HLA$\mathrm{H}$ gene in Italian hemochromatosis patients. Am J Hum Genet 1997; 60: 828-382.

26 Estivill X, Fortina P, Surrey S: Connexin-26 mutations in sporadic and inherited sensorineural deafness. Lancet 1998; 351: 394-398.

27 Morell RJ, Kim HJ, Hood LJ: Mutations in the connexin 26 gene (GJB2) among Ashkenazi Jews with nonsyndromic recessive deafness. NEJM 1998; 339: 1500-1505.

28 Sanguedolce LA, Chan V, McKenzie S, Surrey S, Fortina P, Graves D: Fundamental studies of DNA adsorption and hybridization on solid surfaces. In: Dinh SM, De Nuzzio JD, Comfort AR (eds). Intelligent Materials for Controlled-Release Technologies, vol 728. American Chemical Society: Washington, DC, 1999, pp 190-204.

29 Graves D, Su H-J, McKenzie S, Surrey S, Fortina P: System for preparing microhybridization arrays on glass slides. Anal Chem 1998; 70: 5085-5092.

30 Ross P, Hall L, Smirnov I, Haff L: High level multiplex genotyping by MALDI-TOF mass spectrometry. Nat Biotechnol 1998; 16: 1347-1351.

31 Amersham Life Science: Multicolor fluorescent reagents and directly labeled biologicals. Application note 1995.

32 Prober JM, Trainor GL, Dam RJ et al: A system for rapid DNA sequencing with fluorescent chain-terminating dideoxynucleotides. Science 1987; 238: 336-341.

33 Dubiley S, Kirillov E, Mirzabekov A: Polymorphism analysis and gene detection by minisequencing on an array of gel-immobilized primers. Nucleic Acids Res 1999; 27: el9 i-vi.

34 Southern E, Mir K, Shchepinov M: Molecular interactions on microarrays. Nat Genet 1999; 21(suppl 1): 5-9.

35 Beier M, Hoheisel JD: Versatile derivatisation of solid support media for covalent bonding on DNA-microchip. Nucleic Acids Res 1999; 27: 1970-1977.

36 Kurg A, Tollett JJ, Shan A, Roa BB, Richards CS, Nye SH, Pirrung A, Metspalu A, Shumaker JM: Detection of recurrent mutations in BRCA1 and BRCA2 genes by enzyme-enhanced DNA chips. Am J Hum Genet 1997; 61: A68.

37 Tollett JJ, Kurg A, Shah A, Roa BB, Richards CS, NyeSH, Pirrung M, Metspalu A, Shumaker JM: A chip-based DNA sequence analysis of exons5-9 of the p53 gene. Am J Hum Genet 1997; 61: A1322. 
38 Tollett JJ, Liles LA, Teske JP, Warren JK, Staub RW: The APEX SNP identity chip and the INDENTIPLEX ${ }^{\mathrm{TM}} \mathrm{STR}$ identity testing system. Am J Hum Genet 1999; 65: A2334.

39 Head SR, Rogers J-H, Parikh K et al: Nested genetic bit analysis $\mathrm{N}$-GBA for mutation detection in the p53 tumor suppressor gene. Nucleic Acids Res 1997; 25: 5065-5071.
40 Tonisson N, Kurg A, Lohmussaar E, Metspalu A: Arrayed primer extension on the DNA chip: method and applications. In: Schena M (ed). Microarray Biochip Technology. BioTechniques Books: Natick, MA, 2000, pp 247-263. 